\title{
Evolution of the fusion cross section for light systems at intermediate energies
}

P. Lautesse ${ }^{1 \text { a }}$, L. Nalpas ${ }^{2}$, R. Dayras ${ }^{2}$, M.F. Rivet ${ }^{3}$, M. Pârlog ${ }^{1,4}$, E. Bisquer $^{1}$, B. Borderie ${ }^{3}$, R. Bougault ${ }^{5}$, P. Buchet ${ }^{2}$, J.L. Charvet ${ }^{2}$, A. Chbihi ${ }^{6}$, M. Colonna ${ }^{7}$, A. Demeyer ${ }^{1}$, P. Désesquelles ${ }^{3}$, J.D. Frankland ${ }^{6}$, E. Galichet ${ }^{3,8}$, E. Gerlic ${ }^{1}$, D.C.R. Guinet ${ }^{1}$, R. Legrain ${ }^{2 b}$, N. Le Neindre ${ }^{3}$, O. Lopez ${ }^{5}$, L. Manduci ${ }^{5}$, A.M. Maskay ${ }^{1}$, G. Noguère ${ }^{2}$, E. Rosato ${ }^{9}$, R. Roy ${ }^{10}$, C. Schmitt ${ }^{1}$, M. Stern ${ }^{1}$, B. Tamain ${ }^{5}$, E. Vient ${ }^{5}$, M. Vigilante ${ }^{9}$, C. Volant ${ }^{2}$, and J.P. Wieleczko ${ }^{6}$ (INDRA Collaboration)

1 Institut de Physique Nucléaire, IN2P3-CNRS et Université, F-69622 Villeurbanne cedex, France.

2 DAPNIA/SPhN, CEA/Saclay, F-91191 Gif sur Yvette cedex, France.

3 Institut de Physique Nucléaire, IN2P3-CNRS, F-91406 Orsay cedex, France

4 National Institute for Physics and Nuclear Engineering, RO-76900 Bucharest-Măgurele, Romania.

5 LPC, IN2P3-CNRS, ENSICAEN et Université, F-14050 Caen cedex, France.

6 GANIL, CEA et IN2P3-CNRS, B.P. 55027, F-14076 Caen cedex 5, France.

7 Laboratorio Nazionale del Sud, Viale Andrea Doria, I-95129 Catania, Italy

8 Conservatoire National des Arts et Métiers, F-75141 Paris cedex 03, France.

9 Dipartimento di Scienze Fisiche e Sezione INFN, Universitá di Napoli "Federico II", I80126 Napoli, Italy.

10 Département de physique, Université Laval, Québec, Canada G1K 7P4

April 7, 2006

\begin{abstract}
Ar}+\mathrm{Ni}$ and $\mathrm{Ni}+\mathrm{Ni}$ collisions are investigated between 32 and around $100 \mathrm{~A} \mathrm{MeV}$ incident energy with the $4 \pi$ multidetector INDRA. Fusion cross sections are found to decrease from $\sim 180 \mathrm{mb}$ at $32 A$ $\mathrm{MeV}$ to zero above $50 \mathrm{~A} \mathrm{MeV}$. Other experimental results, for light systems, are compared. Moreover, theoretical works are discussed and fusion cross sections, calculated from two dynamical simulations based on nuclear Boltzmann equation (Boltzmann-Nordheim-Vlasov and Landau-Vlasov models), are also compared to experimental results.
\end{abstract}

PACS. 2 5.70.-z Low and intermediate energy heavy-ion reactions -2 5.70.Jj Fusion and fusion-fission reactions

\section{Introduction}

Fusion processes have been the subject of numerous experimental and theoretical works for many years. Complete fusion is the dominant process in nuclear collisions between medium mass ions near the Coulomb barrier. Above $\sim 8-10 \mathrm{~A} \mathrm{MeV}$, incomplete fusion (IF) sets in, which is generally characterized, for non symmetric systems, through the recoil velocity of evaporation residues or of fissioning nuclei differing from the center of mass velocity $[1,2]$. In this process some nucleons do not enter the compound nucleus and escape as free nucleons or light clusters. It was shown that nucleons may escape if the velocity in the center of mass framework of the nucleus they belong to is larger than a given threshold $(\sim 0.06 \mathrm{c})$ [1]; thus at the lower energies, preequilibrium nucleons originate from the lighter nucleus. Fusion processes were reported to vanish around 35-40 A MeV for Ar-induced reactions [3-

\footnotetext{
a $e$-mail: lautesse@ipnl.in2p3.fr

b deceased
}

7] but persist to much higher energies for very asymmetric collisions, e.g. $\mathrm{C}$ or $\mathrm{N}$ impinging on heavy targets [8, 9]. Different explanations were proposed for the extinction of fusion. It may be due to entrance channel effects, in connection with the viscosity or the stopping power of nuclear matter $[10,11]$ : with increasing energy, some transparency may appear. At rather low energies, a critical angular momentum could limit the fusion processes [12]. At higher energies a limit may appear in the excitation energy which can be supported by a nucleus, either in the total energy [13], or in the energy per nucleon $[14,15]$. The latter can be related to a limiting temperature for nuclei as derived from Hartree-Fock calculations [16]. Note that in this case, one would deal with a limit imposed by the exit channel looked at, namely fusion-evaporation. Multifragmentation becomes dominant when the limiting temperature is reached.

In this work we report on fusion cross sections measured for light systems at energies between 32 and around $100 \mathrm{~A} \mathrm{MeV}$, with the help of the $4 \pi$ array INDRA. With $4 \pi$ devices such as INDRA which detect all charged re- 
action products, it becomes possible to identify and separate fusion events from binary dissipative events which are overwhelming at intermediate energies. For slightly asymmetric systems, identifying a residue is not sufficient to characterize fusion at high energy, as its mass (charge) is smaller than those of the projectile and/or the target [15]. Analysis of the global properties of the emitted products becomes necessary to qualify the type of reaction. Single and multiple fragment exit channels are taken into account in the following.

\section{Experiments and event selection}

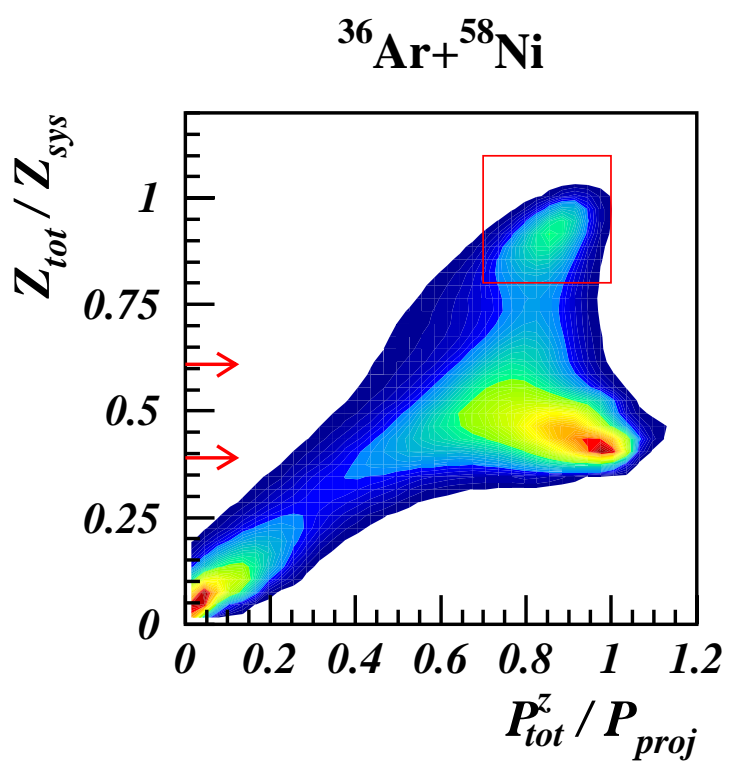

Fig. 1. Total reduced charge $Z_{t o t} / Z_{\text {sys }}$ versus total reduced parallel momentum $P_{\text {tot }}^{z} / P_{\text {proj }}$ measured by INDRA for the $(32 \mathrm{~A} \mathrm{MeV}) \mathrm{Ar}+\mathrm{Ni}$ collisions. $Z_{\text {sys }}$ and $P_{\text {proj }}$ are respectively the total charge of the system and the projectile momentum. The arrows indicate respectively the projectile and the target atomic numbers. The fusion events are extracted from the well measured events defined by the rectangle.

Both symmetric $\left({ }^{58} \mathrm{Ni}+{ }^{58} \mathrm{Ni}\right)$ and asymmetric $\left({ }^{36} \mathrm{Ar}+{ }^{58} \mathrm{Ni}\right.$ systems have been studied. The ${ }^{36} \mathrm{Ar}$ and ${ }^{58} \mathrm{Ni}$ beams were delivered by the Ganil facility at similar bombarding energies $32,40,52,63,74,84$ and $95 \mathrm{~A} \mathrm{MeV}$ (respectively 32 , $40,52,63,74,80$ and $90 \mathrm{~A} \mathrm{MeV}$ for the Ni beam). Thin self supporting ${ }^{58} \mathrm{Ni}$ targets were used with typical thickness of $190 \mu \mathrm{g} / \mathrm{cm}^{2}$. The beam intensity of $3-410^{7}$ ions per second allowed to keep multiple interaction probability below $10^{-4}$. The charged reaction products were detected by INDRA using a minimum biased trigger based on multiplicity $M$ : at least 4 modules fired for $\mathrm{Ni}+\mathrm{Ni}$ at all energies and the highest energies (above $80 \mathrm{~A} \mathrm{MeV}$ ) for $\mathrm{Ar}+\mathrm{Ni}$; at least 3 for the lower Ar energies. Such a trigger eliminates the most peripheral collisions. The hydrogen

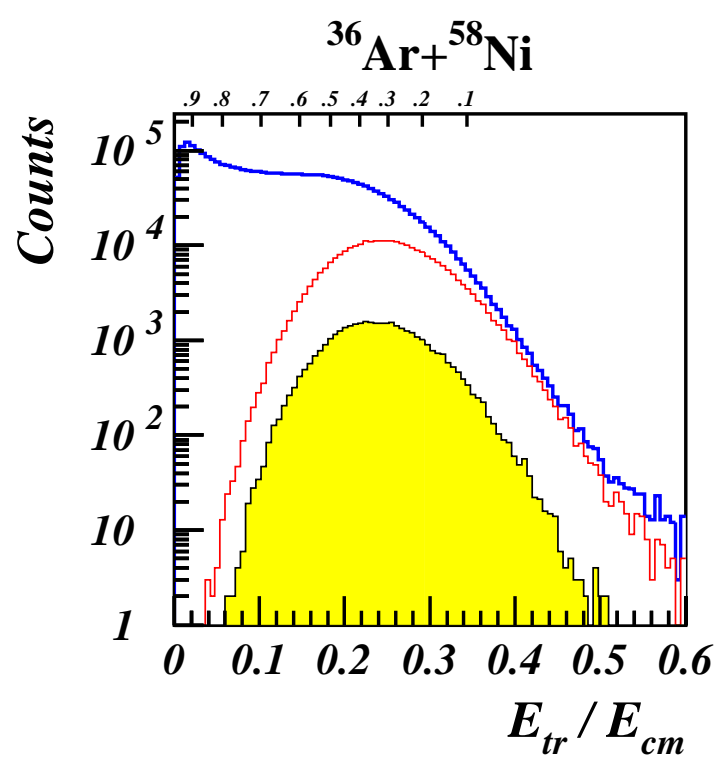

Fig. 2. Transverse energy distributions for all detected events (thick histogram), well measured events (thin histogram) and one-fragment channel (filled histogram) measured in $(32 \mathrm{~A} \mathrm{MeV}) \mathrm{Ar}+\mathrm{Ni}$ reactions. On the top axis, a reduced impact parameter scale $b / b_{\max }$ is given $\left(b_{\max }=9.8 \mathrm{fm}\right)$.

and helium isotopes are fully identified between $3^{\circ}$ and $176^{\circ}$. The charge of the fragments $(Z \geq 3)$ is measured for all the covered energy domain up to the compound nucleus charge with energy thresholds close to $1 A \mathrm{MeV}$ [17]. Notice that backward ionization chambers were not yet installed for the runs performed with Ar beams leading to a fragment identification from $2^{\circ}$ to $88^{\circ}$.

For the forthcoming analyses on both systems, only well measured events are considered for which at least $80 \%$ of the initial charge is detected and the total collected linear momentum is at least equal to $70 \%$ of the beam momentum. An example of these selected events is indicated by the rectangle in Fig. 1 for the ${ }^{36} \mathrm{Ar}+{ }^{58} \mathrm{Ni}$ system at $32 \mathrm{~A} \mathrm{MeV}$ (see [18] for the ${ }^{58} \mathrm{Ni}+{ }^{58} \mathrm{Ni}$ system at $32 \mathrm{~A} \mathrm{MeV}$ ). They represent $10 \%-13 \%$ of the collected events at all bombarding energies and correspond mainly to central collisions $[19,20]$.

The total transverse energy $\left(E_{t r}=\sum_{i} E_{i} \sin ^{2} \theta_{i}\right)$ carried out by the charged products is used to measure the violence of the collisions (Fig. 2 and Fig. 3). The polar angle $\theta_{i}$ is the angle between the beam axis and the velocity direction of the detected product, $i, E_{i}$ being its kinetic energy. A reduced scale of impact parameter $\left(b / b_{\max }\right)$ is obtained by summing the transverse energy distribution using the relation:

$$
\frac{b}{b_{\max }}=\sqrt{K \frac{\int_{E_{t r}}^{\infty} \frac{d \sigma}{d E} d E}{\int_{0}^{\infty} \frac{d \sigma}{d E} d E}} .
$$

The upper limit of the impact parameter scale, $b_{\max }$, is obtained through the relation $\sigma_{R}=\pi b_{\max }^{2}$ from the reaction cross section $\sigma_{R}$ calculated following the prescription 


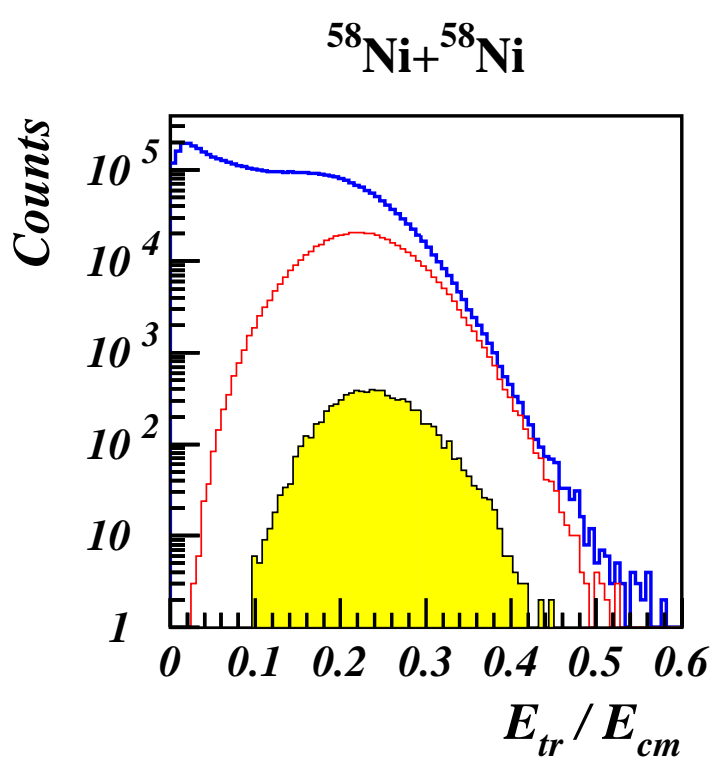

Fig. 3. Transverse energy distributions for all detected events (thick histogram), well measured events (thin histogram) and one-fragment channel (filled histogram) measured in $(32 \mathrm{~A} \mathrm{MeV}) \mathrm{Ni}+\mathrm{Ni}$ reactions.

of [21]. The normalisation factor, $K=\sigma_{\text {exp }} / \sigma_{R}$, takes into account the experimental acceptance of INDRA and the value of the minimum biased trigger [19] which lead to the rejection of the most peripheral collisions. $K \approx 0.8$ for $\mathrm{Ar}+\mathrm{Ni}$ whatever the incident energy. For $\mathrm{Ni}+\mathrm{Ni}, K$ decreases from 0.65 to 0.55 between 32 and $74 A \mathrm{MeV}$. Note that we have verified that the experimental reaction cross sections deduced from events recorded with trigger multiplicity $M \geq 1$, are compatible with the reaction cross sections $\sigma_{R}$ deduced from [21].

The transverse energy distribution of well measured events is broad and covers the tail of the distribution of all detected events corresponding to the most dissipative collisions, namely impact parameter below 3-4 fm depending on the incident energy. The filled histogram corresponds to the transverse energy distribution of events which are good candidates for the fusion process, exhibiting only one fragment $(Z \geq 3)$ in the exit channel. These events are also associated with a broad range of energy dissipation. Their relative abundance is much smaller for the $\mathrm{Ni}+\mathrm{Ni}$ system (see Fig. 3).

\subsection{The Ar+Ni system}

Analyses of the ${ }^{36} \mathrm{Ar}+{ }^{58} \mathrm{Ni}$ collisions from 32 to $95 \mathrm{~A} \mathrm{MeV}$ bombarding energies have pointed out the predominance of binary processes characterized by the presence of projectile and target residues in the exit channel [22]. Their velocities are close to the initial projectile and target velocities. Nevertheless heavy residues are observed (Fig. 4) at the lowest incident energies which suggest the persistence of a fusion process in these collisions.
The mean fragment $(Z \geq 3)$ multiplicity observed for well measured events is $2.4 \pm 1$, independently of the incident energy, with a most likely value of 2 [23]. In order to extract a first evaluation of the fusion cross section, we have considered events which comprise only one fragment with $Z \geq 3$. Characteristics of the residue and the emission patterns of light particles emitted in coincidence for several incident energies $(32,40,52,74$ and $95 A \mathrm{MeV})$ are shown in Figs. 4 and 5. The residue charge versus rapidity distributions (Fig. 4) are peaked slightly below the center of mass rapidity at $32 \mathrm{~A} \mathrm{MeV}$ incident energy, as expected for an incomplete fusion process. This scheme holds up to $52 \mathrm{~A} \mathrm{MeV}$, whereas two components are observed beyond this energy. The transition between these two regimes seems to occur around $50 \mathrm{~A} \mathrm{MeV}$ bombarding energy. The light particle invariant cross section plots (Fig. 5) confirm these features. At $32 \mathrm{~A} \mathrm{MeV}$ they show clearly an emitting source located at the residue velocity. However a fast component is also observed, corresponding to particles escaping in the forward direction. Thus both the fragment and the light particle emission patterns clearly show transparency effects which increase with the incident energy above $50 \mathrm{~A} \mathrm{MeV}$. The evolution of the fragment pattern with energy agrees with the results of [24], where transparency was termed splintering central collisions.

We will firstly discuss the results at $32 \mathrm{~A} \mathrm{MeV}$, the lowest bombarding energy. We observe residues focused at small angles in the laboratory frame. They have large charges $(<Z\rangle=21 \pm 4)$ and a broad rapidity range $\left(<y_{r}>=<y / y_{\text {proj }}>=0.32 \pm 0.08\right)$, the normalised rapidity of the centre of mass being 0.39 ; the mean and the root mean square deviation are mentioned for each distribution. These residues are associated with a large amount of light charged particles which carry away half of the total charge of the system. These features plead in favour of a large mass transfer from the projectile to the target. The part of the projectile which is not involved in the fusion process is obtained from the anisotropies of the angular distributions of the light charged particles observed in the residue frame at forward angles. It is estimated to 6 units of charge ( $\mathrm{Z}=1,2$ only). Events corresponding to one detected residue represent $13 \%$ of the complete events, namely $2 \%$ of the reaction cross section $(\sim 60 \mathrm{mb})$. Similar features are observed for higher fragment multiplicities; in that case, events were attributed to fusion if no projectile residue (defined as a fragment having a rapidity greater than half that of the projectile) is present and the heaviest fragment has a charge greater than 10. Adding these events, the global incomplete fusion cross section reaches $146 \mathrm{mb}$, which corresponds to less than $5 \%$ of the reaction cross section. This value is corrected for an efficiency which is roughly constant with the incident energy, and estimated to $45 \%$ from calculations with the event generator Simon [25]; this efficiency results from filtering the generated events according to the detector acceptance (including the angular coverage and the energy thresholds for the charge identification) and the selection criteria used to sort the fusion events. In view of the selections applied, 


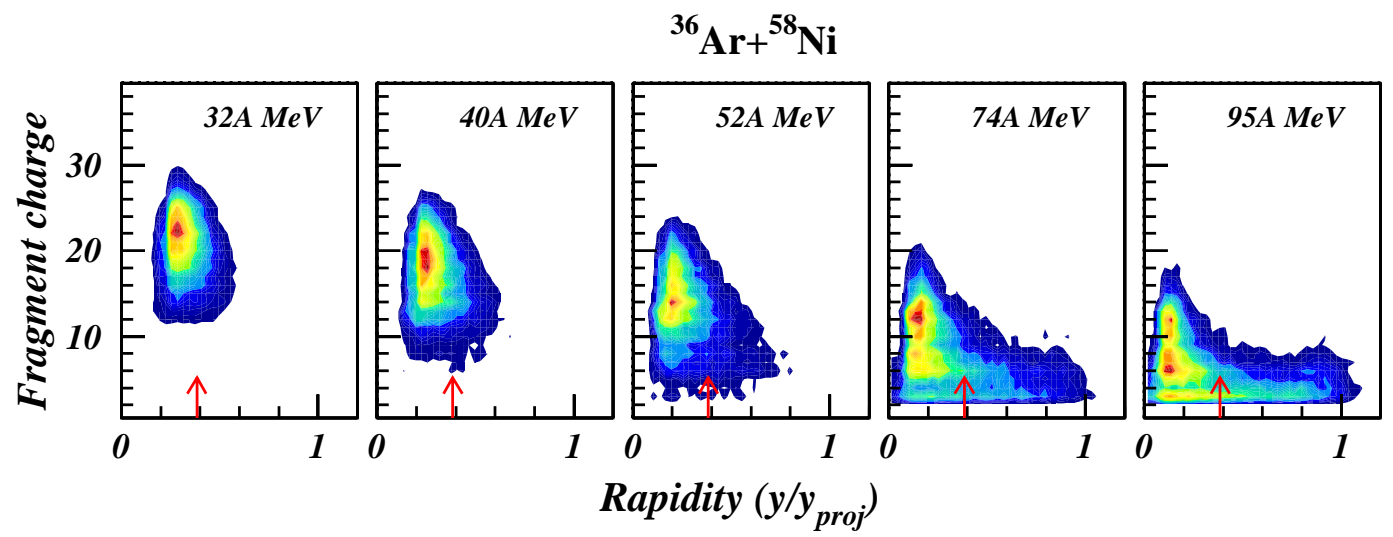

Fig. 4. Charge versus rapidity of the residue observed for the one fragment channel in the Ar+Ni reactions between 32 and $95 \mathrm{~A} \mathrm{MeV}$. The arrows indicate the center of mass rapidity.

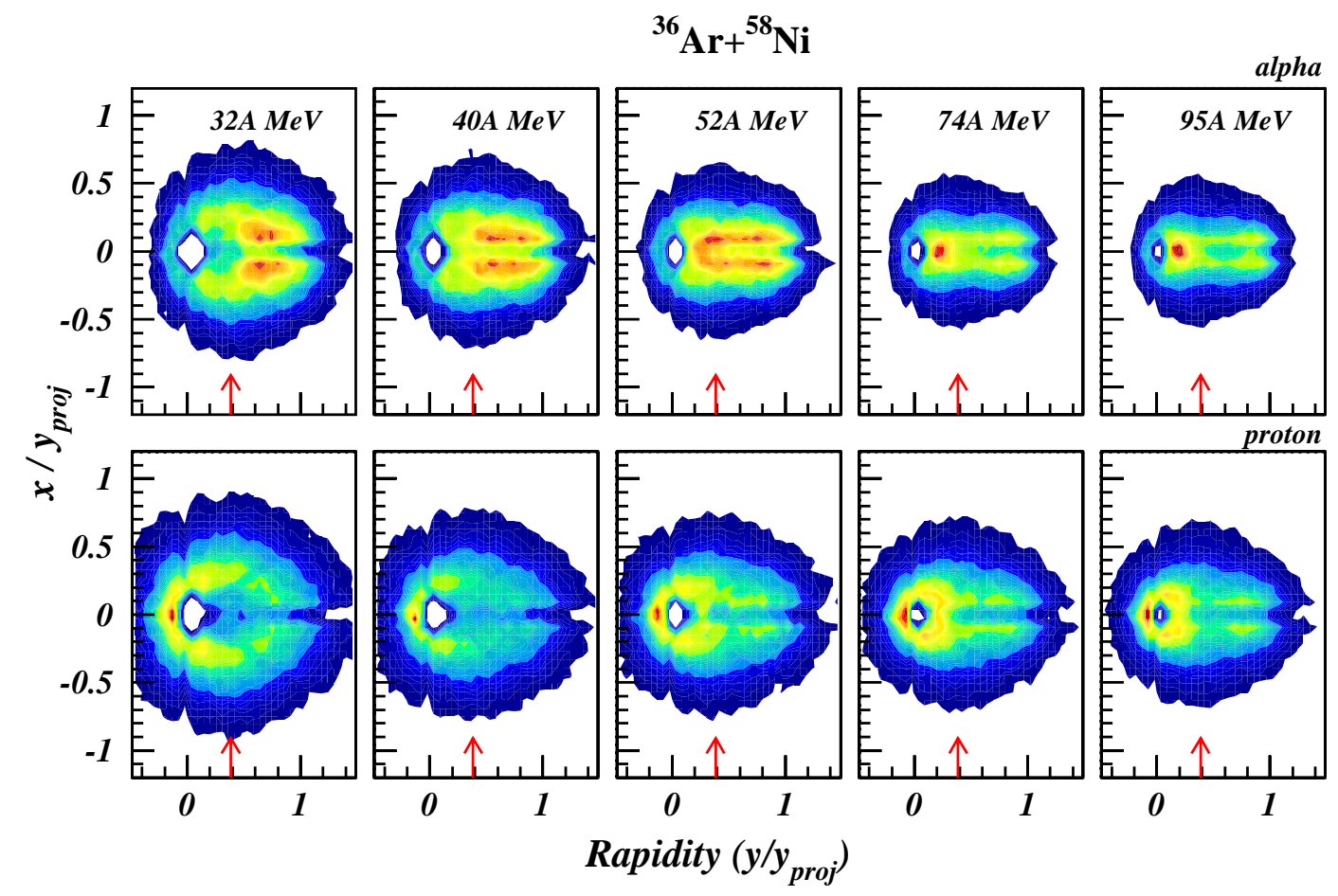

Fig. 5. Invariant cross sections plots for alpha particles (top row) and protons (bottom row) emitted in coincidence with the residue of Fig. 4 . The $x$ variable is the ratio between the transverse momentum and the mass of the particle, $x=p_{t r} / m$. The axes scales are normalised to the projectile rapidity, $y_{\text {proj. }}$. The arrows indicate the center of mass rapidity.

the uncertainty on the cross section is rather large, around $25 \%$ (i.e. $\pm 35 \mathrm{mb}$ ).

The IF cross sections have been extracted in the same way at 40 and $52 \mathrm{AMeV}$ bombarding energies, with larger relative uncertainties and amount respectively to $65 \pm$ $20 \mathrm{mb}$ and $32 \pm 25 \mathrm{mb}$. Above $52 \mathrm{~A} \mathrm{MeV}$ bombarding energy, the velocity of the residue (in the one fragment channel) is farther and farther away from that of the center of mass (Fig. 4). At $95 \mathrm{~A} \mathrm{MeV}$, the slowly recoiling residue is accompanied by fast particles carrying a total charge $\left(Z_{Q P} \sim 17\right)$ close to that of the projectile. The discrimination of the IF events is therefore less and less reliable above $52 \mathrm{AMeV}$. Within the error bars the fusion cross section is compatible with zero.

\subsection{The $\mathrm{Ni}+\mathrm{Ni}$ system}

The previous method based on the identification of a heavy residue in the exit channel has been employed to extract the fusion cross section for the $\mathrm{Ni}+\mathrm{Ni}$ reactions. The most likely fragment $(Z \geq 3)$ multiplicity for the well measured 


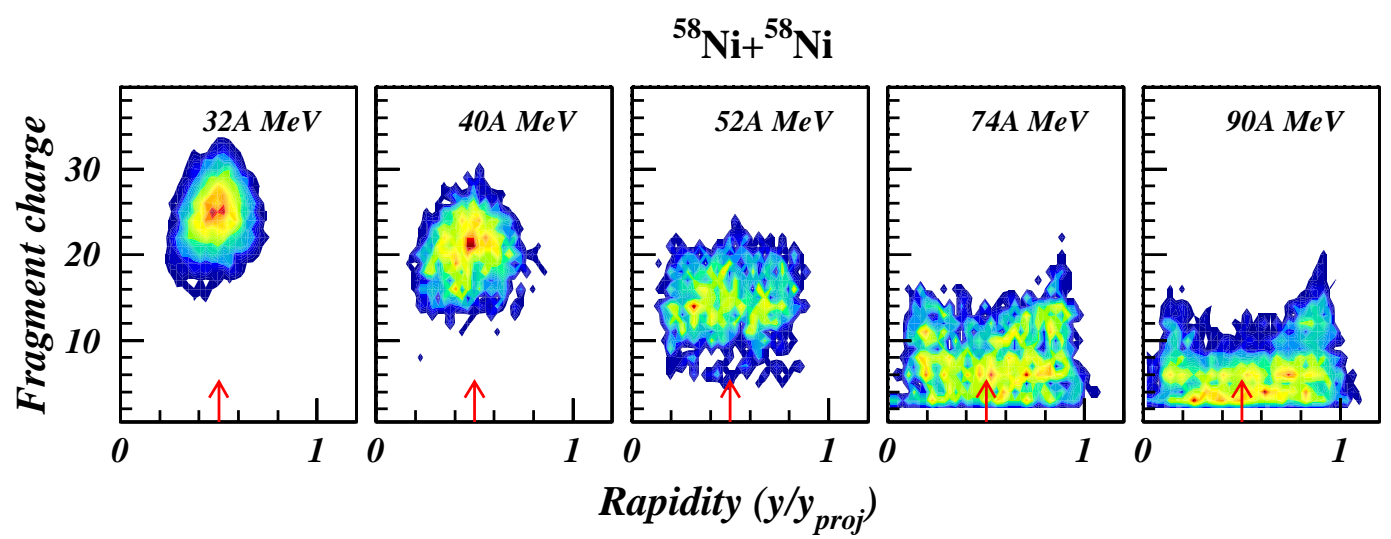

Fig. 6. Charge versus rapidity of the residue observed for the one-fragment channel in the $\mathrm{Ni}+\mathrm{Ni}$ reactions between 32 and $90 \mathrm{~A} \mathrm{MeV}$. The arrows indicate the center of mass rapidity.

events is about 3 , roughly independent of the bombarding energy, and larger by $\sim 1$ unit than that of the $\mathrm{Ar}+\mathrm{Ni}$ reactions. Therefore the one-fragment channel is much less populated in the $\mathrm{Ni}+\mathrm{Ni}$ collisions (see Figs. 2 and 3 ) and represents only $1.6 \%$ of the complete events at $32 \mathrm{~A} \mathrm{MeV}$. Characteristics of this residue are shown in Fig. 6 for several incident energies $(32,40,52,74$ and $90 \mathrm{~A} \mathrm{MeV})$. Similar trends as for the Ar+Ni system are seen: residues having large charge and a rapidity close to the centre-of-mass rapidity are observed below $52 \mathrm{~A} \mathrm{MeV}$. For the high bombarding energies, the rapidity range is broadening up to the projectile and the target rapidities while the size of the residue decreases. Taking advantage of the symmetry of the system, these residues are selected with respect to their charge $(Z>10)$ and to their angular range in the centre-of-mass frame $\left(60^{\circ}<\theta_{c m}<120^{\circ}\right)$. With this selection, a rather clean discrimination of fusion events is achieved and extended to higher fragment multiplicities. The number of fusion events has to be multiplied by a factor of 2 in order to take into account the selected angular range. The values of the fusion cross section thus extracted are compared to the results of a statistical analysis method well adapted to the study of symmetric systems. This Discriminant Analysis method, applied to the mass/kinetic energy quadrimoments, is detailed in [26]. The efficiency of the method for the selection of singlesource events in $(32 \mathrm{~A} \mathrm{MeV}) \mathrm{Ni}+\mathrm{Ni}$ collisions allowed to obtain a reliable separation between single-source events and other event classes. The resulting discriminant variable, so called $d_{625}$, is based on the 625 first quadrimoments [26]. In the present work the determination of the fusion cross section is extended up to $90 \mathrm{~A} \mathrm{MeV}$ beam energy.

The method used to select single source events has been checked using the Simon event generator [26] which simulates statistical decays of single-source or two-source events, depending on the impact parameter in a sharp cut-off prescription. The simulated events were filtered according to the INDRA response function. The event selection procedure is the same for experimental and simulated events at all beam energies. We have checked that the simulated events reproduce quite well the characteristics of the selected experimental ones. The efficiency of the Discriminant Analysis method is characterized by the discriminant power $(0 \leq \lambda \leq 1)$ and the overlap $(0 \leq O \leq 1)$, see definitions in [26]. A perfect discrimination is characterized by $\lambda=1$ and $O=0$. In Table 1 , values obtained from 32 to $74 A \mathrm{MeV}$ are listed.

\begin{tabular}{|c|c|c|}
\hline$E_{\text {incident }}(A \mathrm{MeV})$ & $O$ & $\lambda$ \\
\hline 32 & 0.035 & 0.78 \\
\hline 40 & 0.05 & 0.69 \\
\hline 52 & 0.11 & 0.44 \\
\hline 63.5 & 0.10 & 0.41 \\
\hline 74 & 0.065 & 0.34 \\
\hline
\end{tabular}

Table 1. The overlap $(O)$ and the discriminant power $(\lambda)$ calculated with the Simon code (plus INDRA filter) for $\mathrm{Ni}+\mathrm{Ni}$ system at different incident energies.

At 32 and $40 A \mathrm{MeV}$ the $O$ and $\lambda$ values are quite similar, single-source and two-source events are well separated. At higher energies the discriminant power decreases strongly and the combination of the values of $O$ and $\lambda$ given for $74 \mathrm{AMeV}$ corresponds to the limit of the method. By fitting the experimental discriminant variable distribution with two Gaussians, we can derive the fusion impact parameter probability distribution, allowing to extract the experimental fusion cross section [26].

At $32 \mathrm{~A} \mathrm{MeV}$, after correction for efficiency [26] (which is roughly constant irrespective of the incident energy and estimated to $35 \%$ from calculations with the event generator Simon), the fusion cross section is estimated to $\sigma_{\text {fusion }}$ $\approx 170 \mathrm{mb}$, which still represents about $5 \%$ of the reaction cross section. The fusion cross section falls dramatically for higher energy to be close to $1 \%$ of the reaction cross section at $52 \mathrm{~A} \mathrm{MeV}$ and becomes negligible beyond this 
energy: at $74 \mathrm{~A} \mathrm{MeV}, 82 \mathrm{AMeV}$ incident energies we obtain respectively $10_{-10}^{+20} \mathrm{mb}, 3_{-3}^{+10} \mathrm{mb}$ for the fusion cross section, which vanishes at $90 A \mathrm{MeV}$. Notice that relative error bars increase because single and two-source events are less and less separated (poor discriminant power $\lambda$ ) at high energies.

It was verified that the events selected by the two methods have the same features. The cross sections obtained are displayed in Table 2; they are in good agreement within experimental uncertainties. In the following sections, the weighted average values of the two sets of cross sections will be used (Table 3 ).

\begin{tabular}{|c|c|c|}
\hline$E_{\text {incident }}(A \mathrm{MeV})$ & $\sigma_{N i+N i}^{D A}(\mathrm{mb})$ & $\sigma_{N i+N i}^{H R}(\mathrm{mb})$ \\
\hline 32 & $170 \pm 20$ & $190 \pm 50$ \\
\hline 40 & $90 \pm 20$ & $80 \pm 20$ \\
\hline 52 & $40 \pm 30$ & $25 \pm 20$ \\
\hline 63.5 & $20 \pm 20$ & $7 \pm 7$ \\
\hline
\end{tabular}

Table 2. Ni+Ni fusion cross sections obtained from the Discriminant Analysis method (DA), and the Heavy Residue selection (HR). The values are corrected for experimental efficiency.

\section{Fusion cross sections}

\subsection{INDRA data}

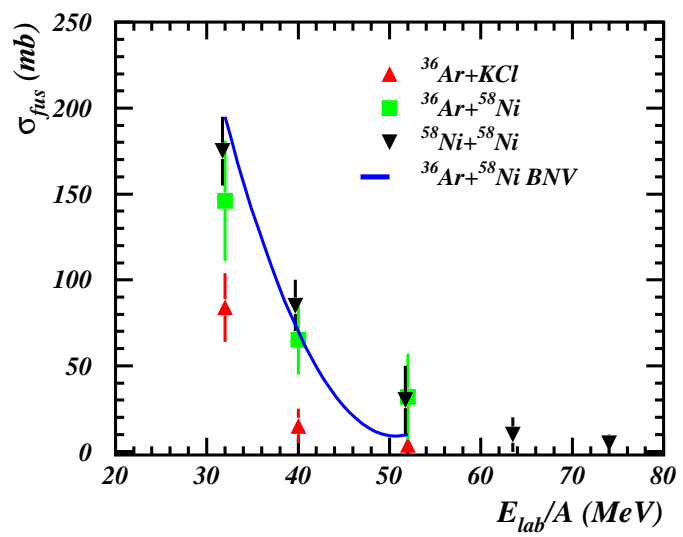

Fig. 7. Fusion cross sections measured for the $\mathrm{Ar}+\mathrm{KCl}$ [27], $\mathrm{Ar}+\mathrm{Ni}$ and $\mathrm{Ni}+\mathrm{Ni}$ systems. For visibility, some points corresponding to 32 and $52 \mathrm{~A} \mathrm{MeV}$ have been slightly shifted. The solid line displays fusion cross sections estimated from BNV simulations for the Ar+Ni system.

Fusion cross sections measured for $\mathrm{Ar}+\mathrm{Ni}$ and $\mathrm{Ni}+\mathrm{Ni}$ are shown in Fig. 7. Results obtained with INDRA using the heavy residue selection for the $\mathrm{Ar}+\mathrm{KCl}$ reaction

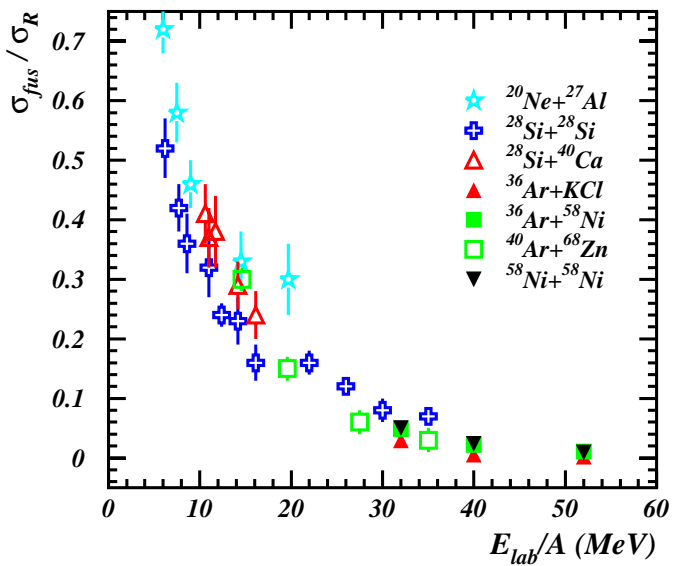

Fig. 8. Fusion cross sections, normalised to the reaction cross section [21], for light systems. Full symbols refer to the INDRA data.

are also displayed [27]. At a given incident energy, fusion cross sections increase with the system mass. A strong decrease of the fusion cross sections is observed beyond $30 \mathrm{AMeV}$, and they seem to vanish above $50 \mathrm{~A} \mathrm{MeV}$, the values quoted at higher energies should be viewed as upper limits.

\subsection{Systematics of fusion for light systems}

Other experimental results found in the literature are listed in Table 3 and displayed in Fig. 8. Data are for $\mathrm{Ne}+\mathrm{Al}$ [28], $\mathrm{Si}+\mathrm{Si}[29,30], \mathrm{Si}+\mathrm{Ca}[31], \mathrm{Ar}+\mathrm{Zn}[4], \mathrm{Ar}+\mathrm{KCl}[27], \mathrm{Ar}+\mathrm{Ni}$ and $\mathrm{Ni}+\mathrm{Ni}$ (this work); the total masses of these systems vary from 47 to 116 . In Fig. 8, fusion cross sections are normalised to reaction cross section, $\sigma_{R}$, which, for consistency, were all calculated following the prescription of [21]. When applying this normalisation, fusion cross sections for all systems gather in a single locus, and display the same behaviour, decreasing from $\sim 70 \%$ of $\sigma_{R}$ at $6 \mathrm{~A} \mathrm{MeV}$ to $10 \%$ around $28-30 \mathrm{~A} \mathrm{MeV}$ and vanishing above $50 A \mathrm{MeV}$. The data obtained with the $4 \pi$ array INDRA thus confirm the previous inclusive measurements existing up to $30 \mathrm{~A} \mathrm{MeV}$. The $4 \pi$ coverage of INDRA allowed to determine the very low values of the fusion cross sections at higher energies. Within the error bars, one does not observe from Fig. 8 any strong effect due to the entrance channel mass asymmetry, $\left(A_{T}-A_{P}\right) /\left(A_{T}+A_{P}\right)$ which varies between 0 and 0.26 for the systems studied, nor to their isospin (1-1.25). The maximal partial wave corresponding to fusion reactions for a given system, in a sharp cut-off approximation, seems to be almost constant between 6 and $25 \mathrm{AMeV}$, and decreases beyond that (Table 3). This may indicate that the concept of critical angular momentum for fusion remains meaningful up to an energy slightly lower than the Fermi energy. The breakdown of this simple concept might therefore indicate the energy where nucleon-nucleon collisions start to significantly compete with mean field. 


\begin{tabular}{|c|c|c|c|c|c|c|c|}
\hline System & Ref & $\begin{array}{c}E_{l a b} \\
\mathrm{MeV}\end{array}$ & $\begin{array}{l}\text { E/A } \\
\mathrm{MeV}\end{array}$ & $\begin{array}{c}\sigma_{f u s} \\
\mathrm{mb}\end{array}$ & $\begin{array}{l}\sigma_{R} \\
\mathrm{mb}\end{array}$ & $\overline{\sigma_{f u s} / \sigma_{R}}$ & $\begin{array}{c}l_{\text {fus }} \\
\hbar\end{array}$ \\
\hline \multirow[t]{5}{*}{${ }^{20} \mathrm{Ne}+{ }^{27} \mathrm{Al}$} & \multirow[t]{5}{*}{ [28] } & 120 & 6.0 & $1122 \pm 60$ & 1559 & $0.72 \pm 0.04$ & $37 \pm 1$ \\
\hline & & 150 & 7.5 & $1000 \pm 80$ & 1738 & $0.58 \pm 0.05$ & $39 \pm 2$ \\
\hline & & 180 & 9.0 & $857 \pm 80$ & 1858 & $0.46 \pm 0.04$ & $39 \pm 2$ \\
\hline & & 290 & 14.5 & $694 \pm 100$ & 2085 & $0.33 \pm 0.05$ & $45 \pm 3$ \\
\hline & & 305 & 19.7 & $612 \pm 120$ & 2040 & $0.30 \pm 0.06$ & $49 \pm 5$ \\
\hline \multirow[t]{11}{*}{${ }^{28} \mathrm{Si}+{ }^{28} \mathrm{Si}$} & \multirow[t]{6}{*}{ [29] } & 174 & 6.2 & $852 \pm 85$ & 1635 & $0.52 \pm 0.05$ & $40 \pm 2$ \\
\hline & & 215 & 7.7 & $788 \pm 78$ & 1857 & $0.42 \pm 0.04$ & $43 \pm 3$ \\
\hline & & 240 & 8.6 & $708 \pm 70$ & 1954 & $0.36 \pm 0.04$ & $43 \pm 3$ \\
\hline & & 309 & 11.0 & $685 \pm 103$ & 2134 & $0.32 \pm 0.05$ & $47 \pm 4$ \\
\hline & & 397 & 14.2 & $525 \pm 79$ & 2269 & $0.23 \pm 0.04$ & $47 \pm 4$ \\
\hline & & 452 & 16.1 & $372 \pm 56$ & 2276 & $0.16 \pm 0.03$ & $42 \pm 3$ \\
\hline & \multirow[t]{5}{*}[30]{} & 347 & 12.4 & $520 \pm 40$ & 2207 & $0.24 \pm 0.02$ & $44 \pm 2$ \\
\hline & & 616 & 22 . & $365 \pm 40$ & 2258 & $0.16 \pm 0.02$ & $49 \pm 3$ \\
\hline & & 728 & 26. & $265 \pm 30$ & 2234 & $0.12 \pm 0.01$ & $45 \pm 3$ \\
\hline & & 840 & 30. & $165 \pm 35$ & 2214 & $0.08 \pm 0.02$ & $38 \pm 4$ \\
\hline & & 980 & 35. & $145 \pm 30$ & 2169 & $0.07 \pm 0.01$ & $39 \pm 4$ \\
\hline \multirow[t]{5}{*}{${ }^{28} \mathrm{Si}+{ }^{40} \mathrm{Ca}$} & \multirow[t]{5}{*}{ [31] } & 298 & 10.6 & $923 \pm 106$ & 2272 & $0.41 \pm 0.05$ & $64 \pm 4$ \\
\hline & & 309 & 11. & $855 \pm 128$ & 2303 & $0.37 \pm 0.05$ & $62 \pm 5$ \\
\hline & & 327 & 11.7 & $898 \pm 143$ & 2352 & $0.38 \pm 0.06$ & $66 \pm 5$ \\
\hline & & 397 & 14.2 & $712 \pm 107$ & 2480 & $0.29 \pm 0.04$ & $65 \pm 5$ \\
\hline & & 452 & 16.1 & $600 \pm 90$ & 2502 & $0.24 \pm 0.04$ & $63 \pm 5$ \\
\hline \multirow[t]{3}{*}{${ }^{36} \mathrm{Ar}+\mathrm{KCl}$} & \multirow[t]{3}{*}{$\overline{[27]}$} & 1137 & 31.6 & $84 \pm 20$ & 2617 & $0.032 \pm 0.008$ & $37 \pm 5$ \\
\hline & & 1441 & 40. & $15 \pm 10$ & 2566 & $0.006 \pm 0.004$ & $17 \pm 6$ \\
\hline & & 1864 & 51.8 & $4 \pm 4$ & 2492 & - & $<10$ \\
\hline \multirow[t]{3}{*}{${ }^{36} \mathrm{Ar}+{ }^{58} \mathrm{Ni}$} & \multirow{3}{*}{$\begin{array}{c}\text { present } \\
\text { work }\end{array}$} & 1137 & 31.6 & $146 \pm 35$ & 3058 & $0.048 \pm 0.011$ & $59 \pm 7$ \\
\hline & & 1441 & 40. & $65 \pm 20$ & 2933 & $0.022 \pm 0.007$ & $44 \pm 7$ \\
\hline & & 1864 & 51.8 & $32 \pm 25$ & 2873 & $0.011 \pm 0.009$ & $35 \pm 14$ \\
\hline \multirow[t]{4}{*}{${ }^{40} \mathrm{Ar}+{ }^{68} \mathrm{Zn}$} & \multirow[t]{4}{*}{ [4] } & 584 & 14.6 & $990 \pm 70$ & 3280 & $0.30 \pm 0.02$ & $118 \pm 4$ \\
\hline & & 784 & 19.6 & $500 \pm 60$ & 3366 & $0.15 \pm 0.02$ & $97 \pm 6$ \\
\hline & & 1102 & 27.5 & $200 \pm 60$ & 3370 & $0.06 \pm 0.02$ & $73 \pm 11$ \\
\hline & & 1400 & 35. & $90 \pm 40$ & 3333 & $0.03 \pm 0.02$ & $55 \pm 12$ \\
\hline \multirow[t]{3}{*}{${ }^{58} \mathrm{Ni}+{ }^{58} \mathrm{Ni}$} & \multirow{3}{*}{$\begin{array}{c}\text { present } \\
\text { work }\end{array}$} & 1856 & 32 & $175 \pm 20$ & 3525 & $0.050 \pm 0.006$ & $84 \pm 5$ \\
\hline & & 2319 & 40. & $85 \pm 15$ & 3501 & $0.024 \pm 0.004$ & $66 \pm 7$ \\
\hline & & 3014 & 52. & $30 \pm 20$ & 3447 & $0.009 \pm 0.006$ & $45 \pm 16$ \\
\hline
\end{tabular}

Table 3. Fusion cross sections published in the literature. $\sigma_{R}$ is calculated as in [21]. $L_{f u s}$ is the maximum partial wave for fusion (in a sharp cut-off approximation) derived from $\sigma_{R}$ and $\sigma_{f u s}$.

\section{Comparison with dynamical simulations}

The occurrence of fusion is linked to the stopping power of nuclear matter, in other words to its viscosity, be it one- or two-body viscosity. In semi-classical simulations, these effects appear through the parametrisation of the mean field and of the nucleon-nucleon cross section, $\sigma_{n n}$. Many studies were done in the last two decades about the respective influence of the stiffness of the equation of state (EOS), of the locality or non locality of the mean field and of $\sigma_{n n}$ on different characteristics of the processes occurring in nuclear collisions, see for instance ref [32-35]. The point here is not to repeat these studies, but, with the supply of new results in the sensitive region where fusion processes disappear, to put still more constraint on the models.

Fusion cross sections are larger, and vanish at higher energies when the stiffness of the EOS, or the number of nucleon-nucleon collisions are increased $[33,36,37]$. We have compared our measurements with published fusion cross sections from two dynamical simulations implemented with different forms of both the mean field and the nucleonnucleon cross section. Both of them use a soft equation of state. Fusion is here defined as reactions leading to the production of a single cluster in the exit channel, at times around 200-300 fm/c. In these light ion reactions at high energy, simulations predict transparency (the incident partners cross each other, giving two clusters in the exit channel).

\subsection{Boltzmann-Nordheim-Vlasov simulations}

$\mathrm{Ar}+\mathrm{Ni}$ reactions were simulated with the Boltzmann-NordheimVlasov (BNV) equation. The density and isospin dependent mean field is approximated with a local Skyrme force, convoluted with a Gaussian to take into account the finite range of the nuclear interaction and some quantum effects $[38,19]$. The chosen parameters correspond to a soft equation of state with a compressibility modulus $\mathrm{K}=200$ 
$\mathrm{MeV}$. The nucleon-nucleon cross section is taken independent of energy and isospin, and equal to $41 \mathrm{mb}$. Fig. 9 displays the evolution of $\mathrm{Ar}+\mathrm{Ni}$ collisions in some chosen cases. In this framework, fusion is observed up to $3 \mathrm{fm}$ at $32 \mathrm{~A} \mathrm{MeV}$, up to $1 \mathrm{fm}$ at $52 \mathrm{~A} \mathrm{MeV}$ and does not occur at higher energies: at $95 \mathrm{AMeV}$, a diluted and expanded system is clearly observed; the projectile has passed through the target and vaporized in the course of the interaction, leading to a spray of light fragments and particles observed in the forward direction. The simulations displayed in Fig. 9 are in full agreement with the experimental pictures of Figs. 4 and 5, where a transparency effect starts to appear at $52 \mathrm{~A} \mathrm{MeV}$ (see subsection 2.1). These calculations also well reproduce the experimental fusion cross sections for the $\mathrm{Ar}+\mathrm{Ni}$ reaction (see Fig. 7).

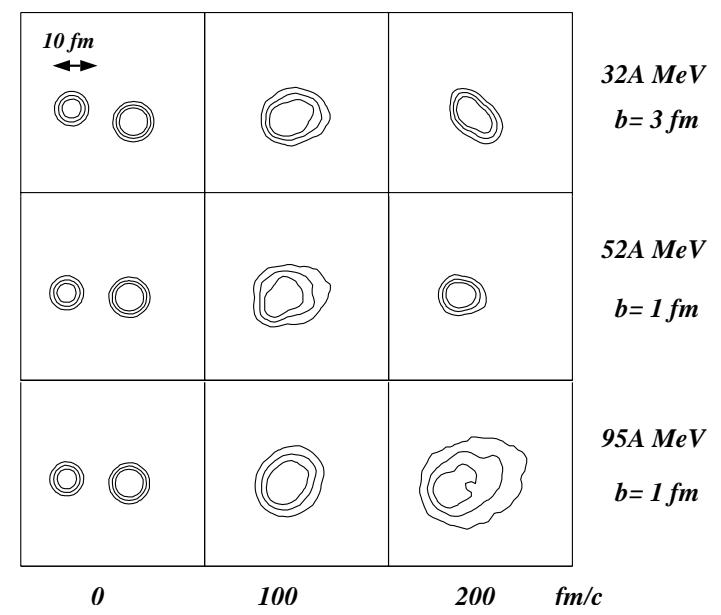

Fig. 9. Evolution with time of the density profiles, in the coordinate space defined by the beam direction (horizontal axis) and the impact parameter (vertical axis), for Ar+Ni collisions at different energies.
This result is clearly inconsistent with the experimental findings. To recover fusion up to $\mathrm{b} \simeq 2.5 \mathrm{fm}$ at $32 \mathrm{~A} \mathrm{MeV}, F$ must be increased up to about 1.5 [37]. The enhancement of $\sigma_{n n}$ around the Fermi energy was indeed suggested in calculations with the Brueckner G-matrix $[39,40]$. It is striking to note that similar higher $\sigma_{n n}$ values were required to reproduce the angular and velocity distributions of fragments produced in dissipative $\mathrm{Ar}+\mathrm{Ag}$ collisions at $27 \mathrm{~A} \mathrm{MeV}$ with the same simulation [41]. These coupled observations on data corresponding to different exit channels reinforce the fact that high $\sigma_{n n}$ values are needed in semi-classical simulations using the Gogny force. This may not be so surprising, as in this case the mean field is more repulsive. Thus to keep the system strongly interacting, a higher residual interaction is required.

To summarize, one should be cautious when extracting values of the in-medium $\sigma_{n n}$ from semi-classical simulations. The present data clearly show that mean field and residual interaction cannot be treated separately, and therefore any value for $\sigma_{n n}$ is valid only in the context of the associated mean-field. Another point to consider is that sharp transitions from fusion to binary collisions when increasing the impact parameter are certainly not realistic, and the two phenomena coexist in central collisions at intermediate energy. This behaviour is fully recognised in simulations as soon as fluctuations are introduced [42]. Fluctuations only influence the dispersion of some variables around the mean field value in stable situations, while in unstable ones they decide which of the possible paths in phase space will be followed (e.g. fusion or binary break-up) [43]. These considerations show first the importance of careful measurements for constraining the parameters of the EOS of nuclei entering in dynamical simulations. The introduction of fluctuations in simulations should also be considered.

\section{Conclusion}

Boltzmann-Uehling-Uhlenbeck simulations with the sameIn this work we have obtained reliable results on the deparameters showed that for the lighter system $\mathrm{Ca}+\mathrm{Ca}$ transparency already occurs at $40 \mathrm{~A} \mathrm{MeV} \mathrm{[33].} \mathrm{This} \mathrm{is} \mathrm{in}$ agreement with the smaller cross sections measured for the $\mathrm{Ar}+\mathrm{KCl}$ system, and indicates that the mass of the system has an important role in the mechanisms occurring in central collisions.

\subsection{Landau-Vlasov simulations}

In Landau-Vlasov dynamical simulations the mean field was implemented by a non local Gogny force. The corresponding EOS is soft, with $\mathrm{K}=228 \mathrm{MeV}$ [32]. In ref [37] the in-medium nucleon-nucleon cross section is parameterized as: $\sigma_{n n}^{m}=F \sigma_{n n}^{f}, \sigma_{n n}^{f}$ being the free nucleon-nucleon cross section, with its isospin and energy dependence and $F$ a corrective constant factor. For $F=1$ fusion disappears at much lower energies than in the previous case, with cross sections strictly equal to zero at $32 \mathrm{AMeV}$ for the three systems $\mathrm{Ar}+\mathrm{Al}, \mathrm{Ar}+\mathrm{Ni}$ and $\mathrm{Ni}+\mathrm{Ni}$ reactions. crease of the fusion cross section in $\mathrm{Ar}+\mathrm{KCl}, \mathrm{Ar}+\mathrm{Ni}$ and $\mathrm{Ni}+\mathrm{Ni}$ reactions from $32 \mathrm{~A} \mathrm{MeV}$ to about $100 \mathrm{~A} \mathrm{MeV}$, measured with the help of the $4 \pi$ multidetector INDRA. The high qualities of the detection device allow to include in fusion cross sections the multifragment channels. The results at $32 \mathrm{~A} \mathrm{MeV} \mathrm{confirm} \mathrm{the} \mathrm{fusion} \mathrm{cross} \mathrm{sections} \mathrm{obtained} \mathrm{so}$ far in inclusive measurements. The new possible extension of measurements for very low cross sections shows that the fusion cross section disappears beyond $40 \mathrm{~A} \mathrm{MeV}$ for $\mathrm{Ar}+\mathrm{KCl}$ system and $50 \mathrm{~A} \mathrm{MeV}$ for $\mathrm{Ar}+\mathrm{Ni}$ and $\mathrm{Ni}+\mathrm{Ni}$ systems. Above this energy a transition between two regimes seems to occur. The complete pattern of all emitted products is fully compatible with a picture where transparency effects are responsible of fusion disappearance.

A systematic investigation of many fusion data between medium mass ions shows that fusion cross sections scale with the reaction cross sections at any given incident energy. No strong effects due either to the entrance channel mass asymmetry $(0-0.26)$ or isospin (1-1.25) are observed. 
The results presented in this paper were confronted to dynamical reaction simulations in order to constrain the stopping parameter (viscosity) of nuclear matter via either the increase of $\sigma_{n n}$ or different mean fields. Furthermore, such experimental results might help to quantify the importance of fluctuations which play, in the Fermi energy region, a non negligible role to describe the reaction process in central collisions.

\section{References}

1. H. Morgenstern, W. Bohne, W. Galster, K. Grabisch and A. Kyanowski, Phys. Rev, Lett. 52, 1104 (1984).

2. M. Fatyga, K. Kwiatkowski, V. E. Viola, C. B. Chitwood, D. J. Fields et al., Phys. Rev. Lett 55, 1376 (1985).

3. H. Nifenecker, J. Blachot, J. Crançon, A. Gizon and A. Lleres, Nucl. Phys. A447, 533 (1985).

4. A. Fahli, J. P. Coffin, G. Guillaume, B. Heusch, F. Jundt et al., Phys. Rev. C34, 161 (1986).

5. M. Conjeaud, S. Harar, M. Mostefai, E. C. Pollacco, C. Volant et al., Phys. Lett. B159, 244 (1985).

6. G. Auger, E. Plagnol, D. Jouan, C. Guet, D. Heuer et al., Phys. Lett B169, 161 (1986).

7. W. G. Gong, C. K. Gelbke, N. Carlin, R. T. de Souza, Y. D. Kim et al., Phys. Lett. B246, 21 (1990).

8. A. A. Sonzogni, A. Elmaani, C. Hyde-Wright, W. Jiang, D. Prindle et al., Phys. Rev. C53, 243 (1996).

9. D. Prindle, A. Elmaani, C. Hyde-Wright, W. Jiang, A. A. Sonzogni et al., Phys. Rev. C57, 1305 (1998).

10. C. Escano-Rodriguez, D. Durand, A. Chbihi, J. D. Frankland, M. L. Begemann-Blaich et al. (INDRA and ALADIN collaborations), Phys. Lett. B, submitted (2005), nucl-ex/0503007.

11. W. Reisdorf, A. Andronic, A. Gobbi, O. Hartmann, N. Herrmann et al. (FOPI Collaboration), Phys. Rev. C92, 232 (2004).

12. R. Bass, Nucl. Phys. A 231, 45 (1974).

13. B. Borderie and M. F. Rivet, Z. Phys. A - Atoms and Nuclei 321, 703 (1985).

14. W. Bohne, H. Morgenstern, K. Grabisch, T. Nakagawa and S. Proschitzki, Phys. Rev. C41, R5 (1990).

15. L. Lassen, P. von Neumann-Cosel, A. Oberstedt and G. Schrieder, Phys. Rev. C 55, 1900 (1997).

16. P. Bonche, S. Levit and D. Vautherin, Nucl. Phys. A436, 265 (1985).

17. J. Pouthas, B. Borderie, R. Dayras, E. Plagnol, M. F. Rivet et al., Nucl. Instr. and Meth. in Phys. Res. A 357, 418 (1995).

18. P. Lautesse, A. M. Maskay, E. Gerlic, P. Désesquelles, J. L. Laville et al. (INDRA collaboration), Phys. Rev. C 71, 034602 (2005).

19. L. Nalpas, thèse de doctorat, Université Paris-XI Orsay (1996), DAPNIA/SPhN-96-02T.

20. P. Buchet, thèse de doctorat, Université de Caen (1999), DAPNIA/SPHN-99-06T.

21. S. Kox, A. Gamp, R. Cherkaoui, A. J. Cole, N. Longequeue et al., Nucl. Phys. A420, 162 (1984).

22. T. Lefort, D. Doré, D. Cussol, Y. Ma, J. Péter et al. (INDRA collaboration), Nucl. Phys. A 662, 397 (2000).

23. L. Nalpas et al., W. Bauer and A. Mignerey, eds., XI Winter Workshop on Nuclear Dynamics, Key West, Florida (Plenum press) (1995), 31, http://hal.ccsd.cnrs.fr/ccsd-00005594.
24. E. Colin, Rulin Sun, N. N. Ajitanand, J. M. Alexander, M. A. Barton et al., Phys. Rev. C 57, 1032 (1998).

25. Anh-Dung Nguyen, thèse de doctorat, Université de Caen (1998), LPCC T 98-02.

26. P. Désesquelles, A. M. Maskay, P. Lautesse, A. Demeyer, E. Gerlic et al. (INDRA collaboration), Phys. Rev. C 62, 024614 (2000).

27. E. Bisquer, thèse de doctorat, Université Claude Bernard Lyon 1 (1996), LYCEN - T 9646.

28. H. Morgenstern, W. Bohne, K. Grabisch and D. Kovar, Phys. Lett. B 113, 463 (1982).

29. M. F. Vineyard, J. S. Bauer, C. H. Gosdin, R. S. Trotter, D. G. Kovar et al., Phys. Rev. C41, 1005 (1990).

30. P. Box, K. A. Griffioen, P. Decowski, T. Bootsma, E. Gierlik et al., Phys. Rev. C50, 934 (1994).

31. M. F. Vineyard, J. S. Bauer, J. F. Crum, C. H. Gosdin, R. S. Trotter et al., Phys. Rev. C41, 1005 (1990).

32. F. Sébille, G. Royer, C. Grégoire, B. Remaud and P. Schuck, Nucl. Phys. A501, 137 (1989).

33. H. M. Xu, W. G. Lynch, P. Danielewicz and G. F. Bertsch, Phys. Rev. Lett 65, 843 (1990).

34. H. M. Xu, Phys. Rev. C 46, R2144 (1992).

35. H. M. Xu, W. G. Lynch and P. Danielewicz, Phys. Rev. C50, 1659 (1994).

36. B. Borderie, M. F. Rivet and L. Tassan-Got, Ann. Phys. Fr. 15, 287 (1990).

37. Z. Basrak and P. Eudes, I. Iori, ed., Proc. XXXVII Int. Winter Meeting on Nuclear Physics, Bormio, Italy (Ricerca scientifica ed educazione permanente) (1999).

38. A. Guarnera, M. Colonna and P. Chomaz, Phys. Lett. B373, 267 (1996).

39. J. Cugnon, A. Lejeune and P. Grangé, Phys. Rev. C35, 861 (1987).

40. A. Bohnet, N. Ohtsuka, J. Aichelin, R. Linden and A. Faessler, Nucl. Phys. A494, 349 (1989).

41. F. Haddad et al., Z. Phys. A - Hadrons and Nuclei 354, 321 (1996).

42. P. Fröbrich and I. I. Gontchar, Phys. Rep. 292, 131 (1998).

43. M. Colonna, M. Di Toro, A. Guarnera, S. Maccarone, M. Zielinska-Pfabé et al., Nucl. Phys. A642, 449 (1998). 